Ciência Florestal, Santa Maria, v. 23, n. 3, p. 417-426, jul.-set., 2013

ISSN 0103-9954

\title{
DUAS NOVAS ESPÉCIES DE Syagrus Mart. (ARECACEAE) PARA O BRASIL
}

\author{
TWO NEW SPECIES OF Syagrus Mart.(ARECACEAE) FROM BRAZIL
}

Kelen Pureza Soares ${ }^{1}$ Ricardo Soares Pimenta ${ }^{2}$ Carlos Alex Guimarães ${ }^{3}$

\section{RESUMO}

Duas novas espécies de Syagrus Mart. (Arecaceae) do Brasil são descritas e ilustradas neste artigo: a primeira, Syagrus pompeoi K. Soares \& R. Pimenta, espécie próxima de Syagrus procumbens Noblick \& Lorenzi, da qual difere pela capacidade de perfilhar, pelo maior comprimento da raque das folhas $(33-50 \mathrm{~cm})$, contendo maior número de pinas de cada lado (8-15), pinas de consistência quase membranosa, com muitas nervuras transversais visíveis e pelo maior comprimento do pedúnculo da inflorescência $(10-18 \mathrm{~cm})$. A segunda, Syagrus santosii K. Soares \& C. A. Guim., espécie próxima de Syagrus schyzophylla (Mart.) Becc., da qual difere por possuir frutos maiores (2,9-4,6 x 2,6-3,8 cm), com o epicarpo e o mesocarpo geralmente fendidos no ápice quando maduros, pelo maior endocarpo (2,7-4,2 x 2,4-3 cm), com endosperma homogêneo, pela maior quantidade de pinas de cada lado da folha e por possuir maiores flores pistiladas.

Palavras-chave: palmeiras; taxonomia; espécie nova; Palmae.

\begin{abstract}
Two new species of Syagrus Mart. (Arecaceae) from Brazil are described and illustrated in this article: first, Syagrus pompeoi K. Soares \& R. Pimenta, a species related to Syagrus procumbens Noblick \& Lorenzi, from which it differs by having a caespitose habit, by the greater length of the rachis of leaves $(33-50 \mathrm{~cm})$, more pinnae on each side (8-15), leaflet almost membranaceous with many transverse ribs visible, as well as longer inflorescence peduncle $(10$ to $18 \mathrm{~cm})$. The second one, Syagrus santosii K. Soares \& C. A. Guim., related to Syagrus schyzophylla (Mart.) Becc., from which it differs by having larger fruit (2.9 to $4.6 \mathrm{x}$ 2.6 to $3.8 \mathrm{~cm})$, with epicarp and mesocarp usually split at the apex when ripe, bigger endocarp $(2.7$ to $4.2 \mathrm{x}$ 2.4 to $3 \mathrm{~cm}$ ) with homogeneous endosperm, greater amount of pinnae on each side of the leaf and larger pistillate flowers.
\end{abstract}

Keywords: palm trees; taxonomy; new species; Palmae.

\section{INTRODUÇÃO}

O gênero Syagrus foi originalmente descrito por Martius (1824), mas nenhuma espécie foi listada no seu trabalho. A primeira espécie descrita para o gênero, Syagrus cocoides Mart., foi estabilizada em 1826 e em anos consequentes, outras foram sendo descritas ou transferidas para este pelo próprio autor (MARTIUS, 1838; 1844), por Wendland (1854), Karsten (1856), Barbosa Rodrigues (1875; 1879; 1888), Beccari (1916), Burret (1932; 1933; 1937; 1940), Bondar (1942), Hawkes (1952), Glassman $(1963 ; 1967 ; 1968 ; 1970 ; 1978)$, Wessels
Boer (1965), Moraes (1996), Noblick (2004; 2004b; 2009) e por Noblick e Lorenzi (2010).

Ao longo dos anos, Syagrus foi segregado em gêneros menores, incorporou os gêneros Butia Becc. e Lytocaryum Toledo e também foi incorporado pelo gênero $\operatorname{Cocos}$ L., na medida em que novas espécies foram descritas e novas revisões foram realizadas. Provavelmente, as quatro espécies presentes no gênero Lytocaryum serão transferidas para Syagrus em futuras revisões, uma vez que a recém-descoberta Lytocaryum itapebiensis Noblick e Lorenzi não apresenta os caracteres morfológicos anteriormente válidos para delimitar o gênero.

1 Engenheiro Florestal, Mestrando do Programa de Pós-graduação em Engenharia Florestal, Centro de Ciências Rurais, Universidade Federal de Santa Maria, Av. Roraima, 1000, CEP 97105-900, Santa Maria (RS).kpsoares@gmail.com

2 Biólogo, Dr., Tropical Plantas e Paisagismo, Av. Major José Levy Sobrinho, 1355, Bairro Jardim Nereide, CEP 13486-190, Limeira (SP).pimenta@fcav.unesp.br

3 Engenheiro Agrônomo, Rua Carneiro da Rocha, 272, CEP 45653-560, Ilhéus (BA).piassava@uol.com.br Recebido para publicação em 20/05/2012 e aceito em 1/4/2013 
Syagrus é um gênero muito variável morfologicamente, quase exclusivo da America do Sul (exceto por Syagrus amara (Jacq.) Mart., que ocorre no Caribe), representado até o momento por 53 espécies, das quais 47 ocorrem no Brasil (NOBLICK, 2010). É composto por palmeiras monoicas, policarpas, de pequeno ou grande porte, solitárias ou entouceiradas, com estipe subterrâneo ou elevado, raramente estolonífero (Syagrus campylospatha Barb. Rodr.), liso ou coberto pelos remanescentes das bainhas das folhas já caídas (DRANSFIELD et al., 2008; NOBLICK, 2010). A maioria das espécies acaulescentes e de pequeno porte está confinada às áreas semiáridas ou de cerrado, enquanto um menor número de espécies de porte arbóreo é comumente encontrado nas áreas tropicais ou subtropicais úmidas, sendo componentes bastante conspícuos em várias formações vegetacionais do Brasil (BARBOSA RODRIGUES, 1903; BONDAR, 1964; DRANSFIELD et al., 2008).

Algumas espécies do gênero são muito valorizadas localmente pelos produtos que delas são retirados, como palmito, amêndoas, polpa dos frutos e folhas para o artesanato; é o caso da guariroba (Syagrus oleracea (Mart.) Becc.), do licuri (Syagrus coronata (Mart.) Becc.) e do gerivá (Syagrus romanzoffiana (Cham.) Glassman) (BONDAR, 1964; NOBLICK, 2010); outras, recentemente, vêm sendo introduzidas com sucesso no paisagismo (NOBLICK, 2010). No entanto, a maioria das espécies encontra-se bastante ameaçada pela expansão da agricultura, especialmente as de pequeno porte, comuns nos cerrados.

\section{MATERIAL E MÉTODO}

A terminologia utilizada para descrever as estruturas vegetativas das espécies seguiu Tomlinson (1990) e Noblick e Lorenzi (2010), que consideram o verdadeiro pecíolo das palmeiras com as margens lisas, nunca cobertas por fibras, sendo que a parte das folhas com margens fibrosas é considerada a bainha. O pseudopecíolo, frequentemente chamado de pecíolo em muitas descrições de palmeiras, inclui a porção arqueada da bainha que se projeta do estipe e possui margens fibrosas ou denteadas e deve ser medido apenas nas folhas mais velhas. Considerouse neste trabalho como "base da bainha" a porção da bainha que se prende ao estipe e que não possui margem denteada na espécie com esta característica. A raque da inflorescência é a medida compreendida entre a inserção da ramificação (ráquila) mais basal até a base da ráquila apical. Uma inflorescência espiciforme é definida como uma ráquila terminal, ou seja, uma inflorescência desprovida de ramificações (TOMLINSON, 1990). A parte onde se inserem as flores de uma inflorescência espiciforme é chamada aqui de parte espigada da inflorescência.

Os dados morfobiométricos, quantitativos e qualitativos, foram tomados in situ de pelo menos 15 palmeiras adultas de cada espécie, incluindo as espécies relacionadas. Apenas os dados referentes ao tamanho do profilo foram baseados em menor número de indivíduos, devido à dificuldade de retirá-lo da planta. Materiais botânicos de todas as espécies foram depositados no Herbário do Departamento de Ciências Florestais da Universidade Federal de Santa Maria (HDCF). Além disso, foram realizadas consultas a materiais bibliográficos, protólogos e ao Herbário Plantarum (HPL).

\section{REDULTADOS E DISCUSÃO}

Syagrus pompeoi K. Soares \& R. Pimenta, sp. nov. (Figura 1).

Palma acaulescens ad $60 \mathrm{~cm}$ alta, solitaria vel caespitosa, cum 1 ad 8 stipibus subterraneis. Folia contemporanea, 3-6 quaeque stipem; rachis longa 33-50, quaeque latus continendo 8-15 pinnas, cum distributione fere regularis longe rachidis et in uno plano insertas; pinnae cum 19-50 x 0,3$1,1 \mathrm{~cm}$, consistentiae coriaceae, fere membranosae, cum transverse nervura sicut striis irregularites dispositis limbo. Inflorescentia interfoliaris, erecta, spiciformis vel raro ramificata, pars inflorescentiae spicata et longa 4,5-7 cm; cum ramificata, ostendit rachillas longas 3-4 cm. Flores staminati cum 9-14 x 4-6 mm, flores pistilati cum 12-19 x 5-7 mm. Fructus 1,8-2,7 x 1,4-2,0 cm; endocarpium 1,4$2,5 \times 0,9-1,4 \mathrm{~cm}$, cavitate interna homogenea, unum semen continens.

Palmeira com até $60 \mathrm{~cm}$ de altura, solitária ou cespitosa, com 1 até 8 estipes subterrâneos de até $4 \mathrm{~cm}$ de diâmetro na base. Folhas contemporâneas, 3 a 6 por estipe, as folhas mais velhas dispostas paralelamente sobre o solo; bainha com 10-18 x 2,6$3,5 \mathrm{~cm}$; pseudopecíolo com margem fibrosa, com 3-6,5 cm de comprimento; pecíolo de margem lisa, com 1,5-7 x 0,3-0,6 cm; raque com 33-50 cm de comprimento contendo (8)-11-15 pinas (folíolos) de cada lado, com distribuição mais ou menos regular ao longo da raque e inseridas em um único plano; pinas de consistência coriácea, quase membranosas, verde-escuras, lineares, com ápice assimétrico, as 

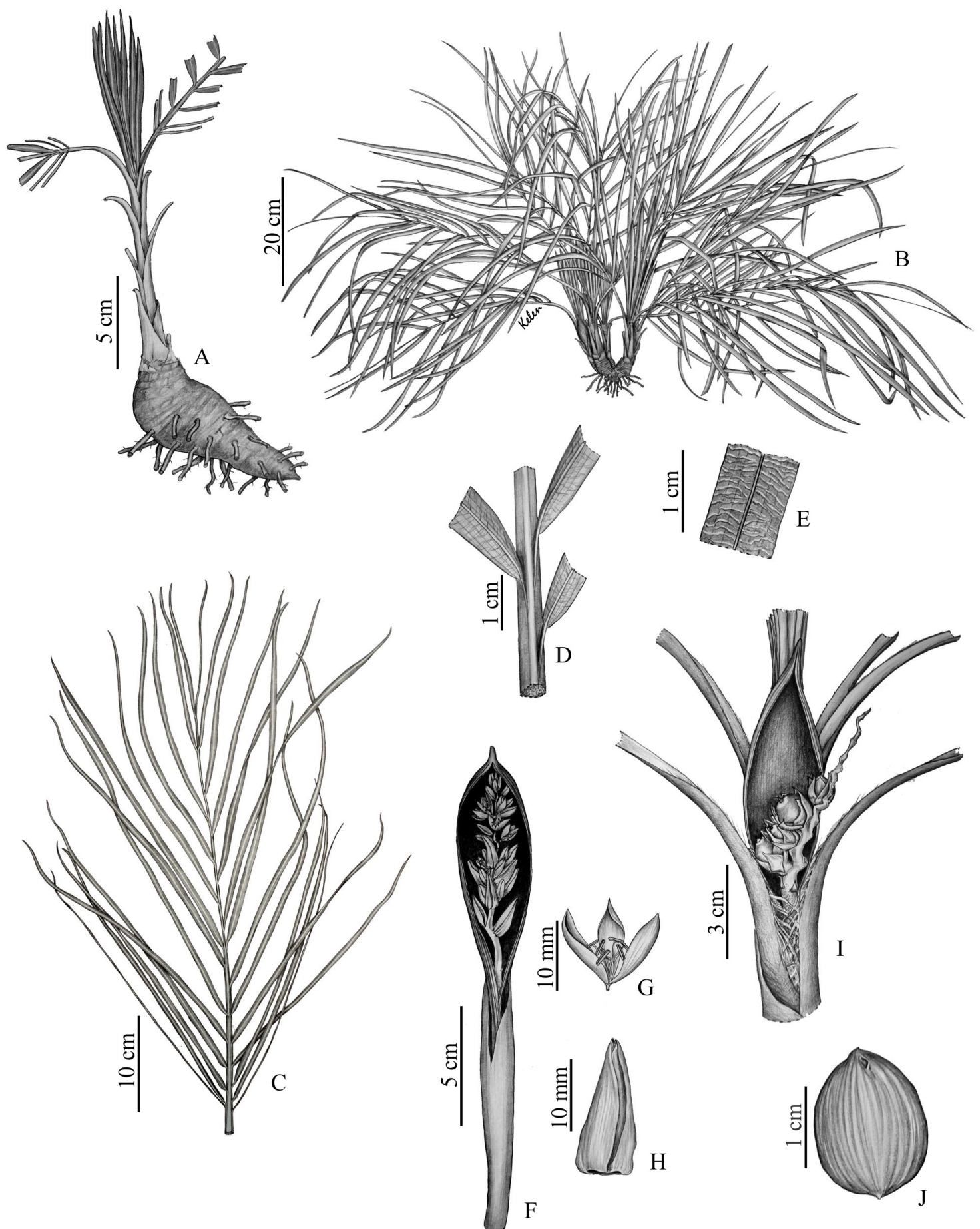

$\mathrm{H}$

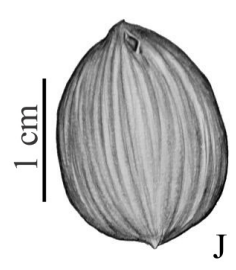

FIGURA 1: Syagrus pompeoi K. Soares \& R. Pimenta. A: hábito mostrando o estipe subterrâneo. B: hábito cespitoso. C: folha. D: detalhe da inserção das pinas na raque. E: detalhe da pina estriada (nervuras transversais) desidratada. F: inflorescência. G: flor estaminada. H: flor pistilada. I: infrutescência. J: endocarpo. (de K. Soares \& R. Pimenta, 14, exceto I: fotografia de R. S. Pimenta. Ilustrado por Kelen P. Soares).

FIGURE 1: Syagrus pompeoi K. Soares \& R. Pimenta. A: Habit showing the subterranean stem. B: caespitose habit. C: leaf. D: detail of the leaflets insertion on the rachis. E: detail of dried striated (transverse ribs) leaflets. F: inflorescence. G: staminate flower. H: pistillate flower. I: infructescence. J: endocarp. (from K. Soares \& R. Pimenta, 14, except I: photography by R. Pimenta. Drawn by Kelen P. Soares). 
da parte basal da raque com $19-43 \times 0,4-0,7 \mathrm{~cm}$, as da parte mediana da raque com $27-50 \times 0,5-1,1 \mathrm{~cm}$, as da extremidade com 19-35 x 0,3-0,6 cm, moderadamente recobertas por uma camada de cera em ambas as faces, mais abundante na face abaxial, que também apresenta mechas de ramento esbranquiçado, estreito e alongado ao longo da nervura principal, bem como, nervuras transversais visíveis dispostas irregularmente no limbo. Inflorescência interfoliar, ereta, espiciforme ou mais raramente ramificada; profilo com $10-13 \times 1,5 \mathrm{~cm}$, lanceolado; pedúnculo com 10-18 x 0,3-0,4 cm; bráctea peduncular sulcada longitudinalmente e levemente coberta por tomento castanho esparso, com 15$23 \mathrm{~cm}$ de comprimento total, parte expandida com 7-10 x 4,5-6 cm, incluindo o bico de 0,5-0,8 cm de comprimento; parte espigada da inflorescência com 4,5-9 cm de comprimento; quando ramificada, apresenta ráquilas com $3-4 \mathrm{~cm}$ de comprimento, a porção com flores pistiladas com 2,5-3,5 cm de comprimento, contendo cerca de 7 flores pistiladas; porção das flores estaminadas com $2-6 \mathrm{~cm}$ de comprimento. Flores estaminadas com 9-14 x 4-6 mm; 3 sépalas com 1-1,5 x 1-1,6 mm, conadas na base; 3 pétalas, com 9-13 x 3,3-5 mm, com pontas agudas e nervuras indistintas; 6 estames com 3,5-5 mm de comprimento, anteras com 3-4 mm. Flores pistiladas piramidais, alongadas, glabras, com 12-19 x 5-7 mm; 3 sépalas imbricadas, com $12-18$ x 5-10 mm, desiguais, com nervuras levemente aparente; 3 pétalas nervadas, com 10-13 x 4-6 mm, lanceoladas, imbricadas na base e valvadas nas extremidades, gineceu com 4-5 x 2-2,5 mm. Frutos elipsoides, amarelos quando maduros, cobertos por uma fina camada tomentosa ferrugínea, com 1,8-2,7 x 1,4-2,0 cm, mesocarpo amarelo, fibroso; endocarpo com 1,4$2,5 \times 0,9-1,4 \mathrm{~cm}$, com a cavidade interna homogênea, contendo uma única semente. Eófilo das plântulas simples.

Tipo: Brasil. Mato Grosso do Sul: Bela Vista, BR 060, $10 \mathrm{Km}$ da cidade de Bela Vista, 07/ VI/2012, fl. fr., K. Soares, R. Pimenta 14 (HDCF 6238).

Distribuição geográfica: espécie conhecida apenas da localidade tipo em Bela Vista, no Mato Grosso do Sul, onde cresce em solo arenoso e rochoso. Trata-se de uma espécie bastante rara, ameaçada pela presença do gado, bem como pela ocupação da área pela agricultura.

Floresce no final do inverno e na primavera e frutifica no verão.

O epíteto específico é uma homenagem à fa- mília Pompeo, de Limeira-SP, em particular a José Pompeo Junior, produtor e colecionador de palmeiras, que há anos vem fomentando as pesquisas de identificação e conservação de palmeiras nativas.

Comentário: Syagrus pompeoi apresenta relação com Syagrus procumbens Noblick \& Lorenzi devido ao estipe subterrâneo, anatomia e disposição das folhas, inflorescência geralmente espigada e pelo tamanho de suas flores; no entanto, pode ser diferenciada desta por apresentar também hábito cespitoso (em Syagrus procumbens somente hábito simples) (Figura 2A e 2B), raque das folhas com 33$50 \mathrm{~cm}$ (em Syagrus procumbens com 2,5-20-(30)), contendo (8)-11-15 pinas de cada lado ((3)-5-8-(11) em Syagrus procumbens), bem como pelas pinas de consistência quase membranosas e com muitas nervuras transversais visíveis (Figura 2C), nas folhas verdes estas nervuras apresentam-se na forma de linhas de cor mais clara, quando a folha é desidratada, elas causam modificações na superfície do limbo apresentando um aspecto estriado, especialmente na parte adaxial (em Syagrus procumbens as pinas são coriáceas e sem nervuras transversais visíveis, mesmo quando desidratadas) (Figura 2D); também apresenta diferenças no comprimento do pedúnculo da inflorescência, que mede 10-18 cm (7-9,5 em Syagrus procumbens) e na bráctea peduncular que é apenas levemente recoberta por um esparso indumento quando jovem (em Syagrus procumbens ela é densamente recoberta por tomento).

Syagrus santosii K. Soares \& C. A. Guim. sp. nov. (Figura 3).

Palma parva vel media, 1,6-4,4 m alta, stipe solitario, generaliter inclinato. Folia, $12-20 \mathrm{~cm}$, contemporanea; pseudo petiolo longo $64-75 \mathrm{~cm}$, cum spinis curvis in tota margine; spinae lignosae, longae ad $2 \mathrm{~cm}$; rachis 160-194 longa, pauca curvata, utraque latus continens 71-90 pinnas; pinnae rigidae, uniforme distribuitae longe rachidis et in uno plano dispositae. Inflorescentia interfoliaris; bractea peduncularis sulcata longitudinaliter; rachis inflorescentiae longa 3848, 43-62 raquilas continendo. Flores masculi 7-10 mm; flores pistilati 7-10 x 6-7 mm. Fructus cum 2,9-4,6 x 2,6-3,8 cm; epicarpium copertum $a b$ tomento ferruginoso in apice fendente, expondo mesocarpium et endocarpium cum maturo est; endocarpium 2,7-3,6 2,4-3,0 cm cum uno semine, endosperma homogeneo.

Palmeira de porte pequeno ou moderado, com 1,6-4,4 m de altura, de estipe solitário, geralmente inclinado, com $0,6-3,5 \mathrm{~m}$ de comprimento e 


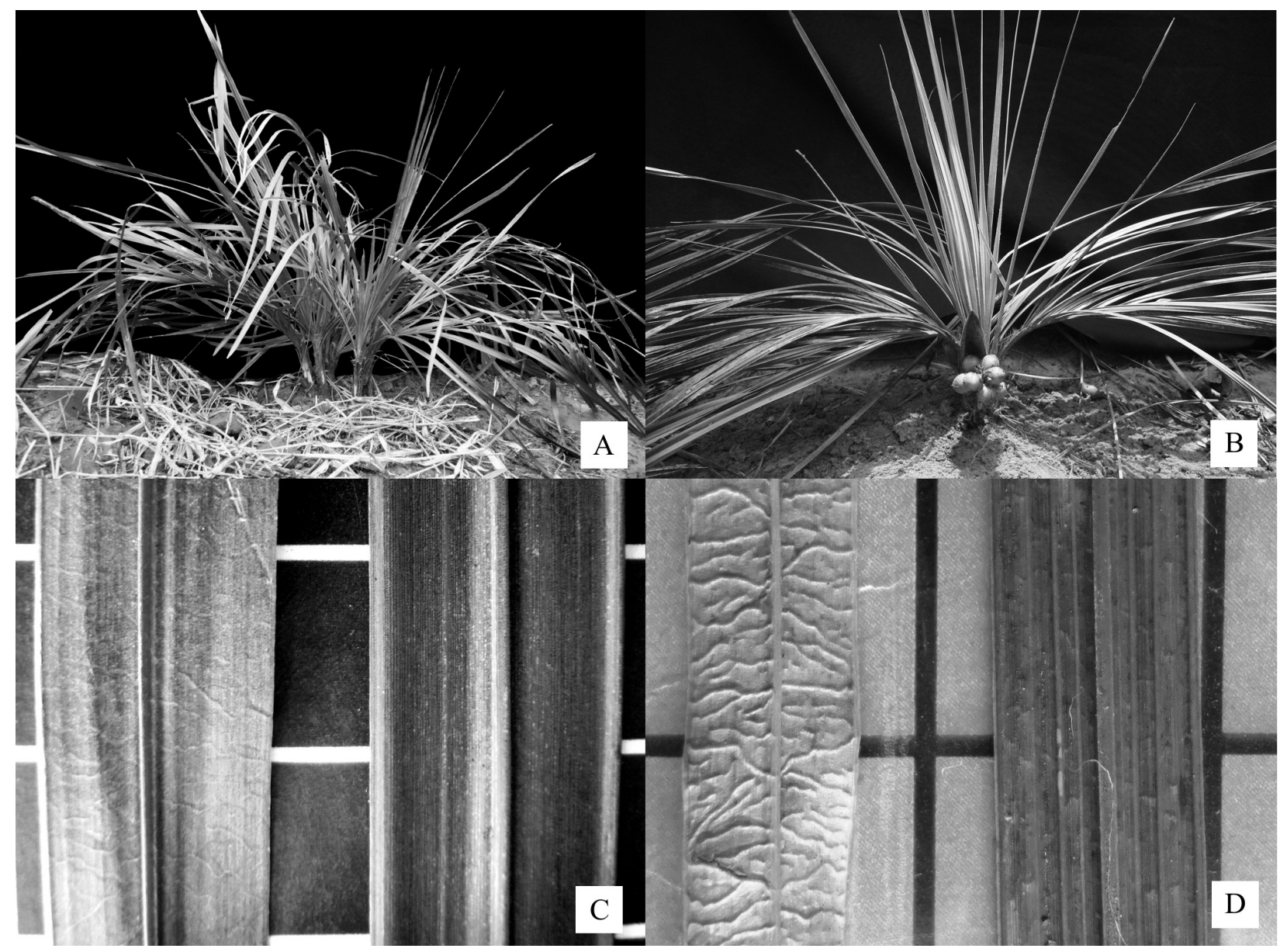

FIGURA 2: A: Syagrus pompeoi K. Soares \& R. Pimenta, hábito cespitoso. B: Syagrus procumbens Noblick \& Lorenzi, hábito. C: detalhe da pina das folhas verdes de Syagrus pompeoi na esquerda e de Syagrus procumbens na direita. D: detalhe da pina das folhas desidratadas de Syagrus pompeoi na esquerda e de Syagrus procumbens na direita.

FIGURE 2: A: Syagrus pompeoi K. Soares \& R. Pimenta, caespitose habit. B: Syagrus procumbens Noblick \& Lorenzi, habit. C: detail of the green leaflet of Syagrus pompeoi on the left and green leaflet of Syagrus procumbens on the right. D: detail of the dried leaflet of Syagrus pompeoi on the left and dried leaflet of Syagrus procumbens on the right.

47-67 cm de circunferência, recoberto pelos remanescentes das bainhas espinescentes de folhas secas ou já caídas, ocasionalmente perdendo este revestimento e deixando cicatrizes que se distanciam uma das outras por $0,5-1,5 \mathrm{~cm}$. Folhas dispostas em espiral ao redor do estipe, 12-20 contemporâneas, pouco curvadas; bainhas com margens quase totalmente cobertas por espinhos, com cerca de $85 \mathrm{~cm}$ de comprimento total, somente a base das bainhas, 15$24 \mathrm{~cm}$ iniciais, possui margens sem espinhos; pseudopecíolo com 64-75 cm de comprimento, inicialmente canaliculado na parte adaxial e arredondado na parte abaxial, armado com espinhos curvados em toda sua margem e revestido por fibras achatadas; espinhos distanciados 1-2 cm um do outro e com até $2 \mathrm{~cm}$ de comprimento, lignosos, sendo a extremida- de destes de apenas fibras decíduas, os maiores espinhos localizados próximos da base das folhas; pecíolo ausente ou com até $2 \mathrm{~cm}$ de comprimento; raque com 160-194 cm de comprimento, pouco curvada, contendo 71-90 pinas de cada lado; pinas rígidas, verdes ou verde-amareladas, brilhantes, com ápice assimétrico, distribuídas uniformemente ao longo da raque e dispostas em um único plano, frequentemente com ramento estreito e alongado ao longo da nervura principal na parte abaxial, as da base das folhas opostas, com 50-58 x 1,2-2,0 cm, as da parte mediana da raque com $46-54$ x 2,0-3,0 cm, as da extremidade da raque opostas ou alternas, com 20-27 x 1,0-1,5 cm. Inflorescência andrógina, interfoliar, envolvida por duas brácteas, a mais interna lenhosa, chamada bráctea peduncular, que protege a inflores- 


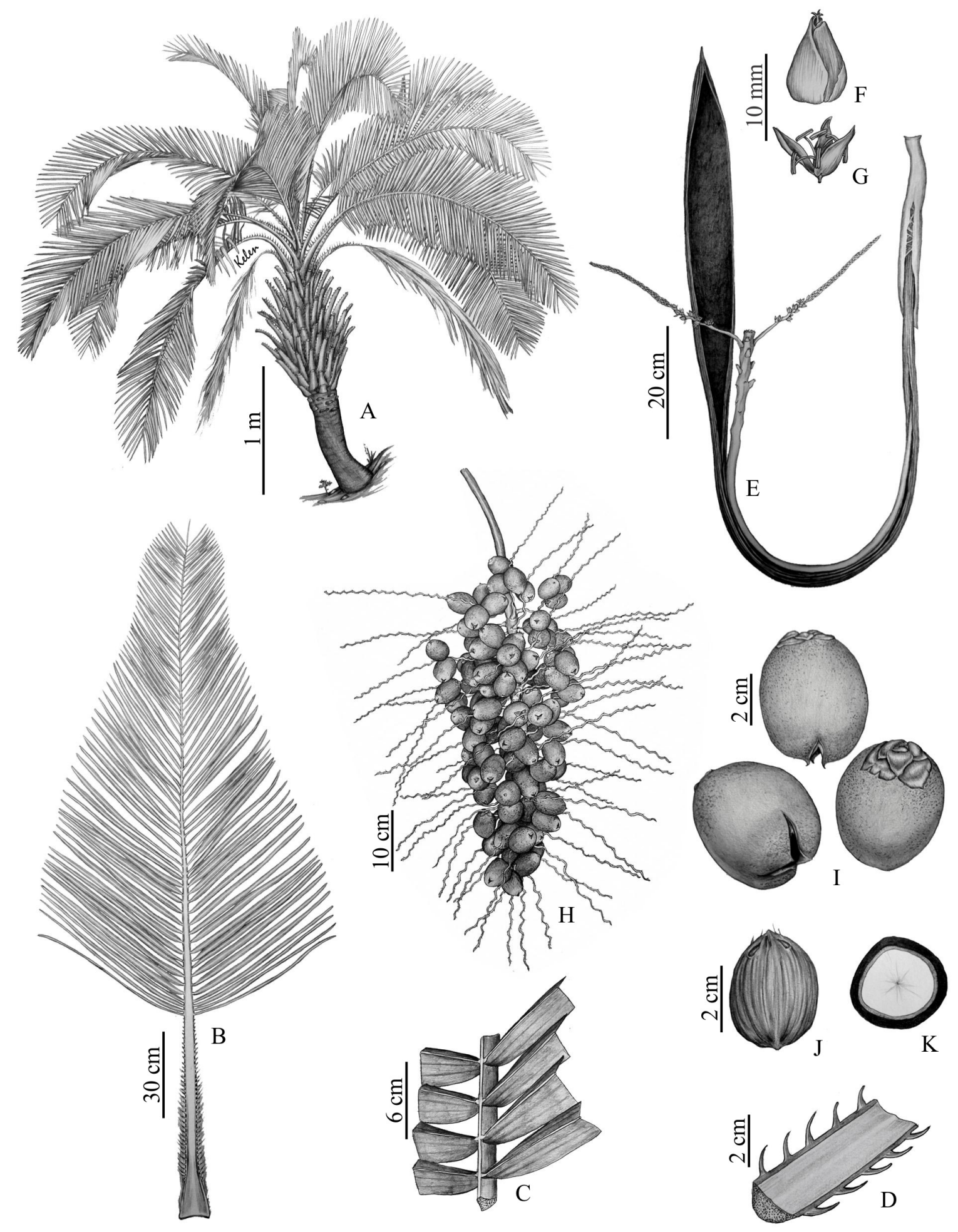

FIGURA 3: Syagrus santosii K. Soares \& C. A. Guim. A: hábito. B: folha. C: detalhe da inserção das pinas na raque. D: pseudopecíolo. E: inflorescência. F: flor pistilada. G: flor estaminada. H: infrutescência. I: frutos. J: endocarpo. K: vista interior do endocarpo mostrando o endosperma homogêneo. (de K. Soares et al., 40. Ilustrado por Kelen P. Soares).

FIGURE 3: Syagrus santosii K. Soares \& C. A. Guim. A: habit. B: leaf. C. detail of the leaflets insertion on the rachis. D: pseudopetiole. E: inflorescence. F: pistillate flower. G: staminate flower. H: infructescence. I: fruits. J: endocarp. K. interior view of the endocarp showing homogeneous endosperm. (from K. Soares et al., 40. Drawn by Kelen P. Soares). 
cência até a antese, contendo sulcos longitudinais, recoberta por tomento ferrugíneo, especialmente na extremidade, com 130-156 cm de comprimento total, a parte expandida com 51-70 x 7,0-8,4 cm e um bico de até $3,5 \mathrm{~cm}$; a bráctea externa fibrosa, lanceolada, chamada profilo, com 26-41 x 4,0-5,2 cm, fica sempre escondida entre as bainhas das folhas; pedúnculo coberto por um indumento esparso, de cor acastanhada quando novo, com 85-120 x 1,2$1,3 \mathrm{~cm}$; raque da inflorescência com $38-48 \mathrm{~cm}$ de comprimento, inicialmente amarelo-esbranquiçada, recoberta por uma camada de cera ferrugínea, tornando-se glabra e esverdeada no período de formação dos frutos, ramificada ao nível de primeira ordem, contendo 43-62 ráquilas; ráquilas da base da inflorescência com $26-34 \mathrm{~cm}$ de comprimento, as da parte mediana com $25-33 \mathrm{~cm}$ de comprimento, as da extremidade com $22-24 \mathrm{~cm}$ de comprimento. Flores unissexuais, amarelas, situadas na porção inferior das ráquilas em grupo de três, com uma flor central feminina ladeada por duas flores masculinas, a porção terminal (superior) das ráquilas apresenta somente flores masculinas; flores estaminadas com 7-10 $\mathrm{mm}$ de comprimento, curto pediceladas na base da ráquila, pedicelo com até $1 \mathrm{~mm}$ de comprimento, as da extremidade sésseis, 3 sépalas triangulares, conadas na base, lobo das sépalas com cerca de $1,5 \mathrm{~mm}$ de comprimento, pétalas em número de 3 , desiguais, valvares, lanceoladas, com pontas agudas, coriáceas, com 6-8 x 1,5-2 mm, estames 6 , distintos, dorsifixos, com 5-6 mm de comprimento, anteras com 2,5-4 mm de comprimento, filetes com 3,5-4 mm de comprimento, pistilódio com menos de $1 \mathrm{~mm}$ de comprimento; flores pistiladas cônicas, sésseis, com 7-10 × 6-7 mm, contendo 3 sépalas e 3 pétalas, desiguais, imbricadas, com nervações quase indistintas, sépalas com 7-9 x 7-10 mm, pétalas glabras, com 6-9 × 7-9 mm, gineceu com 5-7 x 3-4 mm. Frutos elipsoides ou ovoides, com 2,9-4,6 x 2,6-3,8 cm; epicarpo amarelo ou verde-amarelado coberto por um tomento ferrugíneo quando maduro que frequentemente se fende no ápice expondo o mesocarpo e o endocarpo; mesocarpo fibroso, adocicado, de coloração amarelada; endocapo ovoide, ósseo, de cor marrom escuro, superfície exterior quase lisa, com 2,7-4,2 x 2,4-3 cm e 2,5-3 mm de espessura, contendo 3 poros de germinação um pouco depressos no endocarpo e uma única semente com endosperma homogêneo. Eófilo das plântulas simples.

Tipo: Brasil. Bahia: Itapebi, lateral direita da barragem do Rio Jequitinhonha, 1558'21" S,
39³5'36" W. 28/VII/2011, fl. fr.; L. A. Mattos Silva, C. A. Guimarães \& J. L. Paixão 5243 (holótipo HUESC 10000, Isótipos CEPEC, ALCB, RB, R).

Parátipo: Brasil. Bahia: Itapebi, Represa da UHE do Rio Jequitinhonha, 24L 0434042, 8234246. 28/VIII/2012, fl., fr., K. P. Soares; L. C. Assis; C. A. Guimarães; J. E. Santos 40 (HDCF 6276).

Até o momento, a única população desta espécie foi localizada nas margens do Rio Jequitinhonha, no município de Itapebi-BA, onde cresce principalmente num curso de $4 \mathrm{~km}$ na margem direita do rio, em altitudes que variam de 111 a 119 m acima do nível do mar, em terreno com declividade em torno dos $80 \%$, nas áreas de capoeira e com muitos afloramentos de rochas em solo sílico-argiloso. Grande parte da população foi inundada com a construção da barragem da Usina Hidrelétrica de Itapebi. Estima-se que hoje existam cerca de 5.000 espécimes no local, sendo uma espécie rara e bastante ameaçada (Figura 4A). Cresce junto com outras Arecaceae como Allagoptera caudescens (Mart.) Kuntze, Attalea burretiana Bondar e com a naturalizada Elaeis guineensis Jacq.

Floresce e frutifica durante todo ano, com maior abundância nos meses de abril a maio e setembro a outubro.

O epíteto específico é uma homenagem ao parabotânico João Eduardo Santos (Figura 4B), de Itapebi-BA, conhecido por "Joãozinho das Orquídeas", um exímio conhecedor de orquídeas e bromélias e que também descobriu esta palmeira.

Comentário: Martius (1826) descreveu no "Historia Naturalis Palmarum" a espécie Cocos schizophylla Mart., para a região entre Camamú, Salvador e Ilhéus na Bahia, com ilustração do hábito representada na foz do Rio Paraguaçú; segundo o autor, a espécie "cobre densamente as planícies arenosas da região litorânea, bem como nos terrenos litólicos da costa"; nesta obra o autor forneceu uma descrição incompleta do endocarpo, sem mostrar a estrutura interna também nas ilustrações. Barbosa Rodrigues (1891) descreveu uma nova espécie e um novo gênero, Arikuryroba capanemae Barb. Rodr., que apresentava semelhanças com a espécie de Martius, exceto pelo fato de possuir frutos $(2,7 \mathrm{x}$ $2,3 \mathrm{~cm})$ e endocarpos $(2,4 \times 1,7 \mathrm{~cm})$ maiores e com endosperma ruminado, já que, nesta mesma obra, o autor fez também uma descrição completa do fruto e do endocarpo de Cocos schizophylla baseado em observações pessoais, alegando que o mesmo possuiria endosperma homogêneo, endocarpo $(1,6 \mathrm{x}$ $0,8 \mathrm{~cm})$ e frutos menores $(2 \times 1,5 \mathrm{~cm})$; para ele, 


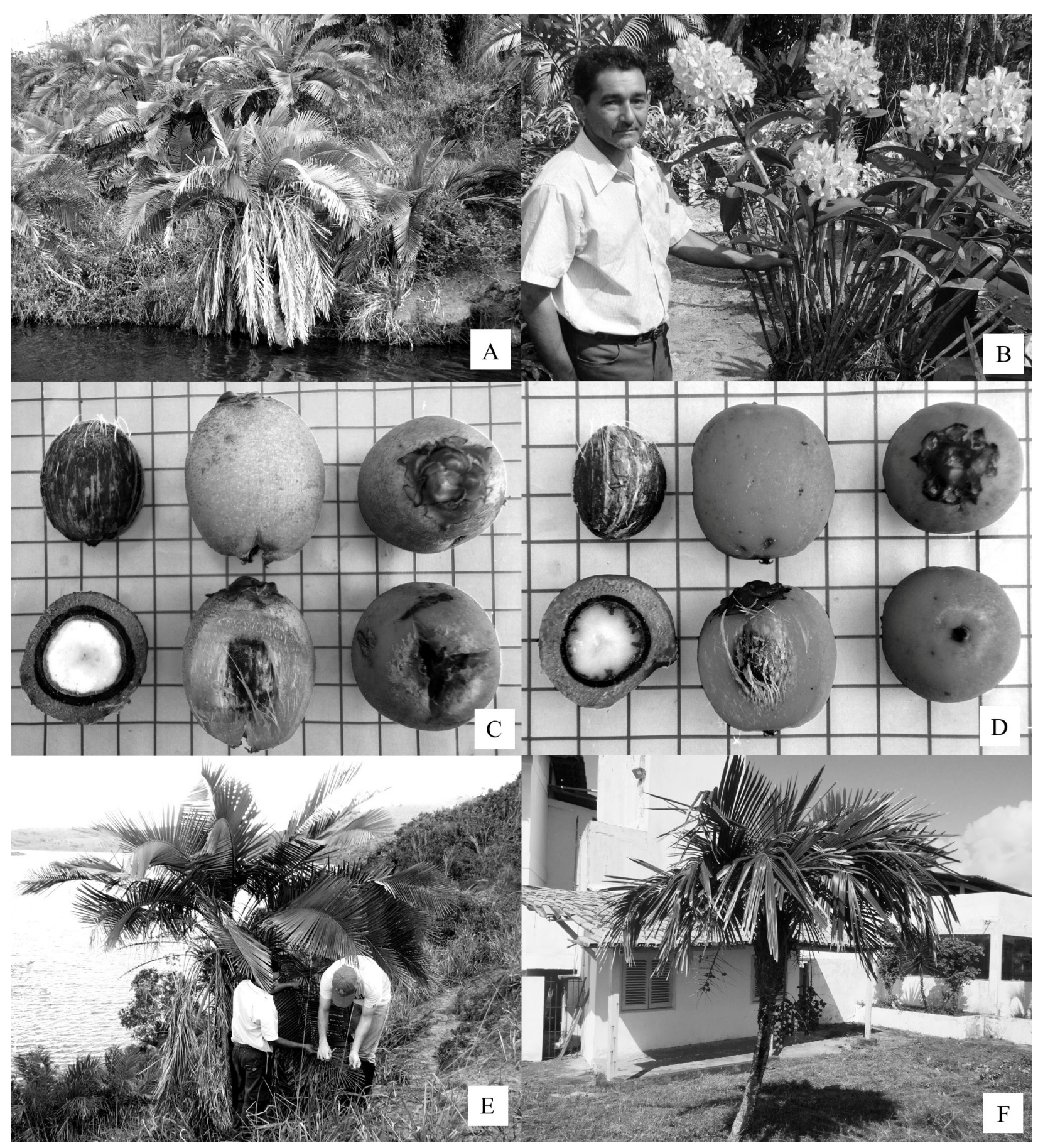

FIGURA 4: A: Syagrus santosii K. Soares \& C. A. Guim. no habitat. B: João Eduardo Santos, descobridor da espécie. C: endocarpo e fruto de Syagrus santosii. D: endocarpo e fruto de Syagrus schyzophylla (Mart.) Glassman. E: hábito de Syagrus santosii. F: hábito de Syagrus schyzophylla.

FIGURE 4: A: Syagrus santosii K. Soares \& C. A. Guim. habitat. B: João Eduardo Santos, species discoverer. C: endocarp and fruit of Syagrus santosii. D: endocarp and fruit of Syagrus schyzophylla (Mart.) Glassman. E: Syagrus santosii habit. F: Syagrus schyzophylla habit.

Cocos schizophylla ocorreria na região de restinga entre Alcobaça e Belmonte - BA.

Glassman (1968) transferiu várias espécies do gênero Cocos e de outros gêneros afins (como Arikuryroba e Arecastrum) para Syagrus, sinonimi- zando a espécie Arikuryroba capanemae à Syagrus schizophylla.

Considerando a característica do endosperma homogêneo, a espécie descrita no presente artigo assemelha-se à Cocos schizophylla sensu 
Martius, exceto pelo fato de possuir frutos e endocarpo muito maiores que o encontrado em ambos os binômios até então descritos. Além disto, em Syagrus santosii o epicarpo e o mesocarpo dos frutos se fendem no ápice em 3 partes quando maduros e são cobertos por um indumento ferrugíneo, medem 2,9-4,6 x 2,6-3,8 cm (2-3,5 x 1,5-2,5 cm em Syagrus schizophylla com epicarpo liso e sem indumento), o endocarpo tem 2,7-4,2 x 2,4-3 cm $(1,8-2,5 \times 1,3-2,2 \mathrm{~cm}$ em Syagrus schizophylla) (Figura 4C e D), apresenta 43-62 ráquilas na sua inflorescência (14-38 em Syagrus schizophylla), flores pistiladas com 7-10 x 6-7 mm (5-8 x 3-6 mm em Syagrus schizophylla), suas folhas patentes têm a raque pouco curvada com 71-90 pinas rígidas de cada lado (em Syagrus schizophylla a raque é curvada com 18-50 pinas de cada lado, geralmente dobradas ou "quebradas") (Figura 4E e 4F). Além disso, a espécie só foi encontrada na região de Itapebi na margem do Rio Jequitinhonha, cerca de $75 \mathrm{~km}$ do litoral.

\section{AGRADECIMENTOS}

Agradecemos à Lucas Coelho de Assis e ao professor Luis Alberto Mattos Silva pela ajuda nas coletas de material botânico; Milton Moreira Silva e Aparecido Donizete Fernandes por ter ajudado a localizar S. pompeoi. À professora Leila Teresinha Maraschin pelas diagnoses em latim.

\section{REFERÊNCIAS BIBLIOGRÁFICAS}

BARBOSA RODRIGUES, J. Enumeratio Palmarum Novarum. Sebastianópolis: Brown \& Evaristo, $1875.43 \mathrm{p}$.

BARBOSA RODRIGUES, J. Palmae Amazonenses

Novae. Vellosia, Manaus, v. 1, p. 33-56, 1888.

BARBOSA RODRIGUES, J. Plantas Novas Cultivadas no Jardim Botânico do Rio de Janeiro. Rio de Janeiro: Leuzinger \& Filhos, 1891. v. 1, p. 5-11.

BARBOSA RODRIGUES, J. Protesto-appendice ao Enumeratio palmarum novarum. Rio de Janeiro: Typografia Nacional, p. 45-46, 1879.

BARBOSA RODRIGUES, J. Sertum Palmarum Brasiliensium. Bruxeles: Imprimerie Typographique Veuve Monnom, 1903. 114 p.

BECCARI, O. Il genere Cocos Linn. E Le palme affini. l'Agricoltura Coloniale, Florença, v. 10, n. 2, p. 489-524, 1916.

BONDAR, G. New palms of Bahia. Field Museum of Natural History Botanical, Chicago, v. 22, p. 457-463, 1942.

BONDAR, G. Palmeiras do Brasil. São Paulo: Instituto de Botânica, 1964. 159 p.

BURRET, M. Die palmengattung Syagrus Mart. Notizblat Botanischen Gartens, Berlin-Dahlen, v. 13, p. 677-698, 1937.

BURRET, M. Palmae neogeae I, Notizblat Botanischen Gartens, Berlin-Dahlen, v. 2, p. 313327, 1932.

BURRET, M. Palmae Neogeae III. Feddes Repertorium, Weinheim, v. 32, p. 102-115, 1933.

BURRET, M. Palmae Neogeae XII. Notizblat Botanischen Gartens, Berlin-Dahlen, v. 15, p. 99108, 1940.

DRANSFIELD, J. et al. Genera Palmarum: the evolution and classification of palms. Londres: Kew Publishing, 2008. 732 p.

GLASSMAN, S. F. A synopsis of the palm genus Syagrus Mart. Fieldiana: botany, Illinois, Chicago, v. 32, n. 15, p. 240, 1970.

GLASSMAN, S. F. New species in the palm genus Syagrus Mart. Fieldiana: botany, Illinois, Chicago, v. 31, n. 9, p. 235-245, 1967.

GLASSMAN, S. F. New species in the palm genus Syagrus Mart., II. Fieldiana: botany, Illinois, Chicago, v. 31, n. 13, p. 285-299, 1968.

GLASSMAN, S. F. New species of Syagrus from the state of Bahia (Brazil), with a revisional study closely related taxa. Phytologia, Nova York, v. 39, n. 6 , p. 401-423, 1978

GLASSMAN, S. F. Nomeclatural changes in the family Palmae. Rhodora, New England, v. 65, p. 259-261, 1963.

HAWKES, A. D., Studies in Brazilian palms: 2. Bondar's species of Brazilian palms. Arquivos de Botânica do Estado de São Paulo, São Paulo, v. 2, n. 6, p. 175-178, 1952.

KARSTEN, H. Plantae columbianae. Linnaea, Berlin, v. 28, 1856. 247 p.

MARTIUS, C. F. P. Historia Naturalis Palmarum, v. 2. Leipzig: T. O. Weigel, p. 129-130, 1826.

MARTIUS, C. F. P. Historia Naturalis Palmarum, v. 3. Leipzig: T. O. Weigel, , p. 290-292, 1838.

MARTIUS, C. F. P. Palmarum familia ejusque genera denuo illustrata. Munich: Typis $M$. Lindauri, v. 18, p. 18. 1824.

MARTIUS, C. F. P. Palmetum Orbignyanum. In: Voyage dans l'Amérique Meridionale, Paris: Veuve Levrault, p. 131-134, 1844.

MORAES, M. Novelties of the genera Parajubaea and Syagrus (Palmae) from Interandean Valleys of 
Bolivia. Novon, Missouri, v. 6, p. 85-92, 1996.

NOBLICK, L. R. Syagrus cearensis, a twinstemmed new palm from Brazil. Palms, Lawrence, Kansas, v. 48, n. 2, p. 70-76, 2004.

NOBLICK, L. R. Syagrus evansiana, a new palm from Minas Gerais, Brazil. Palms, Lawrence, Kansas, v. 53, n. 3, p. 113-118, 2009.

NOBLICK, L. R. Syagrus Mart. In: LORENZI, H.; NOBLICK, L. R.; KAHN, F.; FERREIRA, E. (Eds.). In: Flora Brasileira: Arecaceae (Palmeiras). Nova Odessa-SP: Instituto Plantarum, p. 304-360, 2010.

NOBLICK, L. R. Syagrus vermicularis, a fascinating new palm from northern Brazil. Palms, Lawrence, Kansas, v. 48, n. 3, p. 109-116, 2004b.

NOBLICK, L. R.; LORENZI, H. New Syagrus species from Brazil. Palms, Lawrence, Kansas, v. 54, n. 1, p. 18-42, 2010.

TOMLINSON, P. The Structural Biology of Palms. Oxford: Clarendon Press, 1990. 477 p.

WENDLAND, H. Index palmarum, cyclanthearum, pandanearum, cycadearum, quae in hortis europaeis coluntur. Hannover: Aulica Hahni, 1854. 38 p.

WESSELS BOER, J. G. The indigenous palms of Suriname. Leiden: E. J. Brill, 1965. 172 p. 